\title{
The design and main performance of a hydrogen peroxide/kerosene coaxial-swirl injector in a lab-scale rocket engine
}

\author{
Huixin $\mathrm{Li}^{\mathrm{a}, \mathrm{b}}$, Liang Ye ${ }^{\mathrm{a}, \mathrm{b}}$, Xiaolin Wei ${ }^{\mathrm{a}, \mathrm{b}, *}$, Teng $\mathrm{Li}^{\mathrm{a}}$, Sen $\mathrm{Li}^{\mathrm{a}}$ \\ a State Key Laboratory of High Temperature Gas Dynamics, Institute of Mechanics, Chinese Academy of Sciences, No. 15 Beisihuanxi Road, Beijing, 100190 , China \\ b School of Engineering and Science, University of Chinese Academy of Sciences, 19 A Yuquan Road, Beijing, 100049, China
}

\section{A R T I C L E I N F O}

\section{Article history:}

Received 1 March 2017

Received in revised form 3 August 2017

Accepted 7 September 2017

Available online 11 September 2017

\section{Keywords:}

$\mathrm{H}_{2} \mathrm{O}_{2}$ /kerosene bipropellant

Coaxial-swirl injector

Geometric constant

Performance

\begin{abstract}
A B S T R A C T
Future space missions are considering the use of green space propulsion, and $\mathrm{H}_{2} \mathrm{O}_{2}$ /kerosene bipropellant is one of effective alternatives for rocket propellant. In addition, for a $100-\mathrm{N}$-class thruster of $\mathrm{H}_{2} \mathrm{O}_{2} /$ kerosene, it is important to design the proper injector. In this study, according to different values of the geometric constant $A$ which determines the initial swirl intensity of the injected fluid, five coaxial swirl injectors are designed to check the thruster's performance. From the test and experiment results, it is found that with an increase in $A$, coaxial-swirl injectors can aid in the mixing of oxidant and fuel but increase the average temperature of faceplate, thus significantly reducing the flame length. However, a large or small value of $A$ is not conductive to improve the combustion efficiency. In addition, the pressure oscillation $\left(P_{\text {ins }}\right)$ inside of the chamber gradually becomes intense. Among all cases, Case 3 ( $A=3.0$ ), exhibits optimal performance for a $100-\mathrm{N}$-class $\mathrm{H}_{2} \mathrm{O}_{2} /$ kerosene thruster.
\end{abstract}

(c) 2017 Elsevier Masson SAS. All rights reserved.

\section{Introduction}

With the goal of meeting new requirements for attitude control during spacecraft and satellite missions, green propulsion research studies have been conducted to develop new systems that are safer and easier to handle and use green and nontoxic propellants to achieve high performance at low cost. Thus, a number of the investigated propellants were hydrogen peroxide [1], kerosene, ammonium dinitramide (ADN) [2,3] and hydroxylammonium nitrate (HAN) [4] for thrusters, the Table 1 demonstrates the properties of some green mono-, and bi-propellants. Among these monopropellants, $\mathrm{H}_{2} \mathrm{O}_{2}$ was used as an oxidizer component of bipropellants and as a monopropellant since the early days of rocket development because of its storable characteristic. Hypergolic $\mathrm{H}_{2} \mathrm{O}_{2}$ / kerosene bipropellant is an effective alternative for the present propulsion systems $[10,11]$, in which hydrogen peroxide at a high concentration becomes an oxidizer to kerosene. A propellant based on hydrogen peroxide has a number of advantages, such as high density, zero toxicity, and high specific impulse [12].

The injector design is critical because it controls the performance and heat transfer characteristics of the combustor and determines whether combustion instabilities will occur. A transverse

\footnotetext{
* Corresponding author at: State Key Laboratory of High Temperature Gas Dynamics, Institute of Mechanics, Chinese Academy of Sciences, No. 15 Beisihuanxi Road, Beijing, 100190, China.

E-mail address: xlwei@imech.ac.cn (X.L. Wei).
}

injector is used in a dump-combustor configuration to investigate the autoignition characteristics of JP-8 in decomposed hydrogen peroxide; specifically, fuel-rich autoignition is limited at contraction ratios less than 6 and in the presence of high hydrogen peroxide concentrations [13]. Using numerical simulation, Li et al. [14] found that a $\mathrm{H}_{2} \mathrm{O}_{2} /$ kerosene coaxial shear injector causes the combustion chamber to be divided into the following zones: rapid high-temperature pyrolysis zone, oxidization zone and equilibrium flow zone. In a previous experiment, non-intrusive optical diagnostics was employed to study the combustion behaviors and flame stabilization of a coaxial swirl injector and a coaxial shear injector, including gaseous hydrogen/gaseous oxygen $\left(\mathrm{GO}_{2} / \mathrm{GH}_{2}\right)$ and gaseous methane/gaseous oxygen $\left(\mathrm{GCH}_{4} / \mathrm{GO}_{2}\right)$ [15,16]. The method may also be employed in $\mathrm{H}_{2} \mathrm{O}_{2}$ / kerosene bipropellant.

However, a few challenges still exist in the development of the injector of the $\mathrm{H}_{2} \mathrm{O}_{2}$ / kerosene bipropellant 100 -N-class thruster. The thruster must have a small mass flow rate, low weight and small size, which require the injector and combustor to not only have a small structure but also ensure flow conditions for autoignition and good mixture. In addition, compared with the $\mathrm{N}_{2} \mathrm{O}_{4}$-derivative/ $\mathrm{N}_{2} \mathrm{H}_{4}$-derivative rocket propellant system, the propellant mixture ratio of $\mathrm{H}_{2} \mathrm{O}_{2} /$ kerosene can reach 7.5 [9], thus making it difficult to mix a small amount of fuel with the oxidizer.

To address these challenges, this paper presents a design for coaxial swirl injectors based on the previous injector design achievements $[17,18]$. Hulka et al. $[19,20]$ conducted a series of 
Table 1

The properties of some green propellants.

\begin{tabular}{|c|c|c|c|c|c|c|}
\hline Propellant & & $\begin{array}{l}\text { Toxicity (LD50 } \\
\text { orally } \mathrm{mg} / \mathrm{kg} \text { ) }\end{array}$ & $\begin{array}{l}\text { Mass ratio } \\
\text { (oxidizer/fuel) }\end{array}$ & $\begin{array}{l}\text { Vacuum } \\
I_{s p}(\mathrm{~s})\end{array}$ & $\begin{array}{l}\text { Density } I_{s p} \times 10^{3} \\
\left(\mathrm{~kg} / \mathrm{m}^{3} \cdot \mathrm{s}\right)\end{array}$ & Ref. \\
\hline \multirow[t]{3}{*}{ Monopropellant } & LMP-101 & 1360 rats & - & 246.94 & 350.65 & [5] \\
\hline & HAN (100\%) & 325 rats & - & 217.85 & 398.67 & {$[4]$} \\
\hline & $90 \% \mathrm{H}_{2} \mathrm{O}_{2}$ & 376 rats & - & 170 & 235.96 & {$[1,6]$} \\
\hline \multirow[t]{3}{*}{ Bipropellants } & LOX and & - & 2.74 & 343.57 & 352.45 & {$[7,8]$} \\
\hline & Kerosene & $>5000$ rats & & & & \\
\hline & $\begin{array}{l}90 \% \mathrm{H}_{2} \mathrm{O}_{2} \text { and } \\
\text { Kerosene }\end{array}$ & $\begin{array}{l}376 \text { rats } \\
>5000 \text { rats }\end{array}$ & 7.5 & 290 & 375.37 & {$[7,9]$} \\
\hline
\end{tabular}

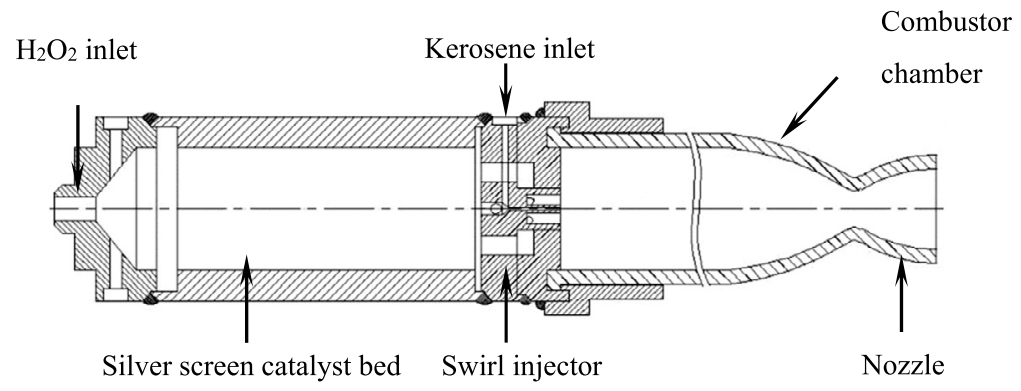

Fig. 1. Schematic of the laboratory rocket engine.

Table 2

The main physical properties of $90 \% \mathrm{H}_{2} \mathrm{O}_{2}$ and kerosene.

\begin{tabular}{llllll}
\hline Propellant & $\begin{array}{l}\text { Temperature } \\
(\mathrm{K})\end{array}$ & $\begin{array}{l}\text { Pressure } \\
(\mathrm{MPa})\end{array}$ & $\begin{array}{l}\text { Density } \\
\left(\mathrm{kg} / \mathrm{m}^{3}\right)\end{array}$ & $\begin{array}{l}\text { Viscosity } \\
(\mathrm{Pa} \cdot \mathrm{s})\end{array}$ & Ref. \\
\hline $90 \% \mathrm{H}_{2} \mathrm{O}_{2}$ & 293 & 0.101 & 1386.7 & $1.25 \times 10^{-3}$ & {$[22]$} \\
Decomposed $\mathrm{H}_{2} \mathrm{O}_{2}$ & 1030 & 2.8 & 7.23 & $3.66 \times 10^{-5}$ & NIST \\
Kerosene & 300 & 2.8 & 780 & $1.21 \times 10^{-3}$ & {$[23]$} \\
\hline
\end{tabular}

cold-flow studies to optimize the design of coaxial swirl injectors for liquid rockets. Compared with jet injectors, the non-uniform mixing of propellants is avoided and the mixing efficiency is significantly improved. In addition, the injector is less susceptible to choking and cavitation [21]. The coaxial swirl injector has good mixing and promotes the reduction of the flame length, both of which improve the thruster's efficiency and decreases the thruster's weight.

\section{Experimental setup}

\subsection{Lab-scale rocket engine configurations}

All tests are conducted in a lab-scale rocket engine system. The rocket engine consists of the following (as shown in Fig. 1): silver screen catalyst bed, injector, combustion chamber and nozzle. In this paper, a previous lab-scale $100-\mathrm{N}$-class engine is employed [14], but the coaxial swirl injector is used. The length and inner diameter of the combustion chamber are $100 \mathrm{~mm}$ and $30 \mathrm{~mm}$, respectively. The diameter of the nozzle throat is $6.8 \mathrm{~mm}$. Because the engine requirement is a $100 \mathrm{~N}$ thrust, through aerodynamics, it is easy to determine that the total mass flow rate is approximately $50 \mathrm{~g} / \mathrm{s}$ and the diameter of nozzle exit is $12.62 \mathrm{~mm}$.

Here, 90\% hydrogen peroxide and kerosene are pressurized with a regulated nitrogen gas manifold system. With a propellant mixture ratio of 7.5 , the mass flow rate of $90 \%$ hydrogen peroxide and kerosene are $43.7 \mathrm{~g} / \mathrm{s}$ and $6.3 \mathrm{~g} / \mathrm{s}$, respectively. In addition, when hydrogen peroxide passes through kerosene, it is decomposed into high-temperature mixture gas of water vapor and oxygen. The temperature reaches $1030 \mathrm{~K}$, and the mass concentrations of $\mathrm{O}_{2}$ and $\mathrm{H}_{2} \mathrm{O}$ are $42.3 \%$ and $57.7 \%$, respectively.

\subsection{Injector design}

Because the mass flow rate of kerosene is too small, kerosene is injected by the jet atomizer in the center, and decomposed hydrogen peroxide is injected via the four swirl injection atomizer in the outer annulus. Thus, the design is divided into two parts: liquid jet injector and gas swirl injector. Table 2 indicates the main physical properties of the propellants used in the design. The properties of decomposed $\mathrm{H}_{2} \mathrm{O}_{2}$ (mass concentration: $42.3 \% \mathrm{O}_{2}, 57.7 \% \mathrm{H}_{2} \mathrm{O}$ ) is calculated by NIST.

\subsubsection{Liquid jet injector}

Usually, the jet injector design is primarily based on conservation of mass and Bernoulli's equation. However, the hydraulic loss coefficient and the injector configuration must be considered in the real injector, which makes the design procedure complicated. Thus, the explicit expression of mass flow rate can be written as

$\dot{m}_{j}=\mu \bullet A_{j} \bullet \sqrt{2 \rho_{k} \Delta p}$

where $\mu$ is defined as the injector flow coefficient, $\rho_{k}$ is the density of kerosene, $\Delta p$ is the pressure drop of injector and $A_{j}$ is the flow area at the injection exit. The flow coefficient is affected by many factors, including the injector configuration, pressure drop, and physical properties of fluid. To make the expanding jet reach the injector wall, the injector passage must be sufficiently long, with a $L_{j} / d_{j}$ greater than 1.5 [17]. China No. 3 aviation kerosene is employed in the experiment. Finally, one obtains

$d_{j} \cong 0.6 \mathrm{~mm}$

In addition, all of the co-axial swirl injectors include the same jet injector in their design. 


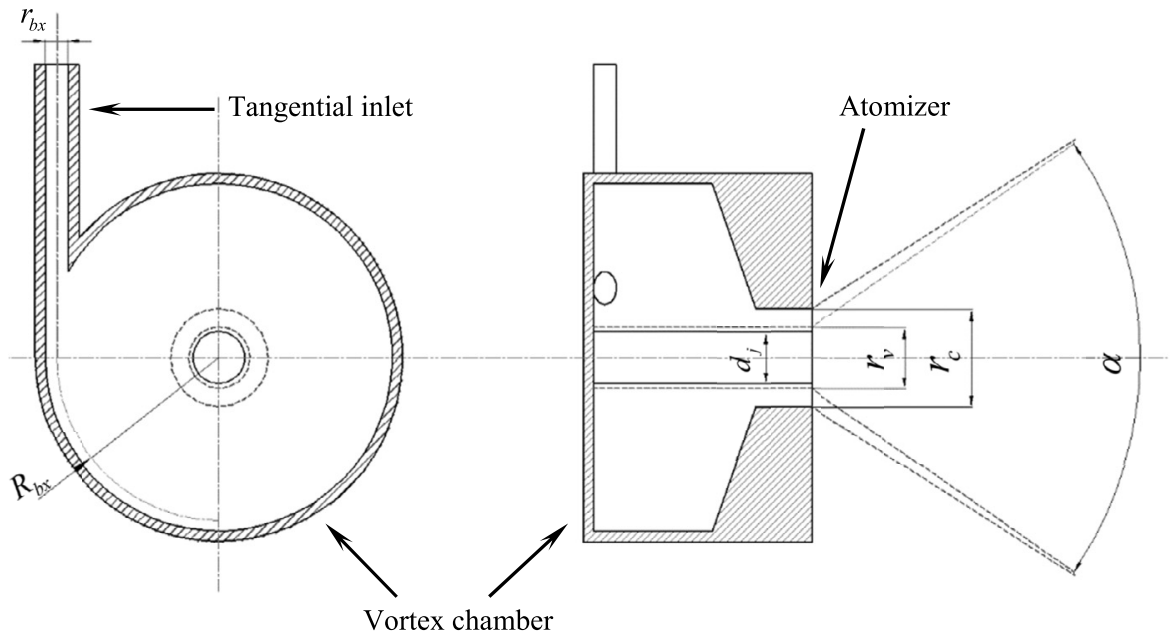

Fig. 2. The physical model of the coaxial swirl injector.

Table 3

Main design parameters of the swirl injector

\begin{tabular}{lllllll}
\hline Case & $A$ & $\mu$ & $\begin{array}{l}\Delta p \\
(\mathrm{MPa})\end{array}$ & $\begin{array}{l}r_{c} \\
(\mathrm{~mm})\end{array}$ & $\begin{array}{l}r_{b x} \\
(\mathrm{~mm})\end{array}$ & $\begin{array}{l}L_{b x} \\
(\mathrm{~mm})\end{array}$ \\
\hline 1 & 6.7 & 0.095 & 0.80 & 8.80 & 1.55 & 8.00 \\
2 & 4.8 & 0.119 & 0.80 & 7.87 & 1.60 & 8.00 \\
3 & 3.0 & 0.168 & 0.80 & 6.62 & 1.66 & 8.00 \\
4 & 1.3 & 0.308 & 0.80 & 4.89 & 1.73 & 8.00 \\
5 & 0.5 & 0.477 & 0.80 & 3.93 & 1.96 & 8.00 \\
\hline
\end{tabular}

\subsubsection{Gas swirl injector}

Compared with the jet injector, the ideal swirl injector design is based on Bernoulli's equation, conservation of mass energy and conservation of momentum. The gas swirl injector has a flow characteristic of center swirl intensity. The oxygen is injected tangentially into the center post of the injector through tangential inlets to create a swirling flow. Thus, the gas swirl injector consists of three major parts: tangential inlets, a vortex chamber, and an atomizer. Fig. 2 shows the physical model of coaxial swirl injector. The swirl injector has an important dimensionless number - the geometric characteristic constant, $A$, which determines the initial swirl intensity of the injected fluid. The constant is defined as

$A=\frac{A_{c} R_{b x}}{A_{i n} r_{c}}=\frac{R_{b x} r_{c}}{n r_{b x}^{2}}$

where $A_{c}$ is the atomizer area, $r_{c}$ is the atomizer radius, $n$ is the number of tangential inlet passages, $A_{i n}$ is the total area of tangential inlets, $r_{b x}$ is the radius of tangential inlets and $R_{b x}$ is the radial location of the tangential inlets. The geometric characteristic constant $A$ can also be expressed by other forms [24]; one of the forms is as follows:

$A=\frac{R_{b x} v_{i n}}{r_{c} v_{a}}$

where $v_{\text {in }}$ is the tangential velocity of the tangential inlet passages and $v_{a}$ is the axial velocity of the injection. Thus, according to the properties of decomposed hydrogen peroxide, using orthogonal experimental design, five swirl injectors are considered; the main design parameters are presented in Table 3. $\mu$ is the flow coefficient, $L_{b x}$ is the passage length of tangential inlet and $\Delta p$ is pressure drop of injector. The details of design procedure can be found in Ref. $[17,24]$.

Fig. 3a indicates the final assembly of the coaxial swirl injector. Decomposed hydrogen peroxide enters six holes and then forms the swirl flow through the four inlet passages of the vortex chamber. Kerosene is injected from the side and forms the jet flow.

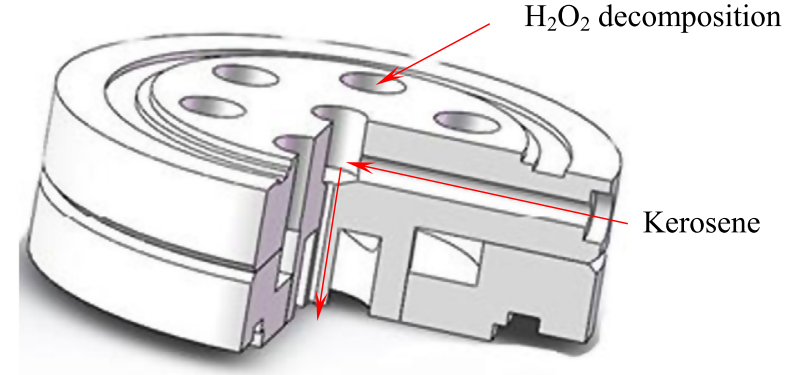

(a)

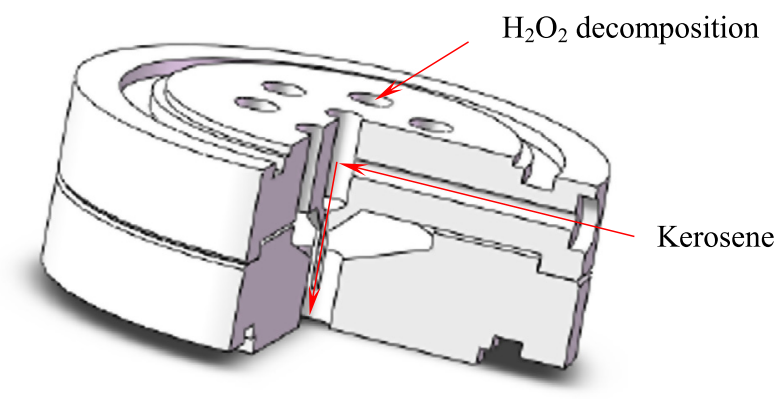

(b)

Fig. 3. (a) The assembly of the coaxial swirl injector. (b) The assembly of the coaxial jet injector.

Next, kerosene and decomposed hydrogen peroxide are mixed in the front of the combustion chamber. And the coaxial jet injector is shown in the Fig. 3b. Decomposed hydrogen peroxide enters six holes and then forms the jet flow through the chamber of the injector.

\section{Simulation model}

\subsection{Physics model}

In the CFD simulation, kerosene injection is substituted by the "Plain Orifice Atomizer" (POA) model and the Discrete Phase Model (DPM). The detailed descriptions of the abovementioned models are given in Ref. [14]. The main parameters used in the POA model are as follows: inner diameter of kerosene tube: $0.6 \mathrm{~mm}$; tube length: $6 \mathrm{~mm}$; and mass-flow rate of kerosene: $6.3 \mathrm{~g} / \mathrm{s}$. A realizable $k-\varepsilon$ turbulence model is employed that exhibits superior per- 


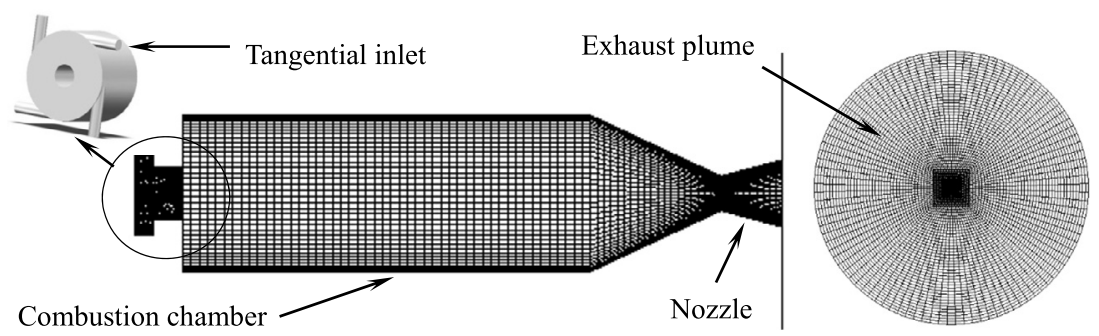

a. Grids of combustion chamber.

b. Right elevation of mesh

Fig. 4. Geometric model and grids.

formance for flows involving rotation and boundary layers under strong adverse pressure gradients, separation, and recirculation.

The equation of state employed in the study is the PengRobinson (PR) cubic equation, which is mainly applied to the following: multicomponent mixtures, high-pressure phase equilibria, supercritical fluids, and reservoir fluids [25]. The equation is given by

$P=\frac{R T}{V-b}-\frac{a}{V^{2}+2 b V-b^{2}}$

where $R$ is the universal gas constant and $V$ is the molar volume. The coefficients $(a$ and $b$ ) represent the attraction and repulsion effect among molecules, respectively.

The combustion in the liquid rocket belongs to a typically turbulent flamelet regime. In all cases, the Reynolds number of cold state kerosene in injector is about $1.053 \times 10^{4}$; thus, a flamelet approach is used to model the non-premixed combustion. Moreover, non-equilibrium effects and a PDF-treatment are considered in the turbulent combustion. Many research works have investigated the mechanism of China No. 3 aviation kerosene [26,27]. In this study, a high-temperature combustion skeletal mechanism [27] containing 79 species and 330 reactions is employed in the thermochemistry calculations.

\subsection{Geometric model, mesh generation and boundary conditions}

The geometric model of injector and grids are shown in Fig. 4, where the mesh of exhaust plume is not shown but Fig. $4 \mathrm{~b}$ is right elevation of exhaust plume. Four tangential inlets represent the tangential inlet passages of the vortex chamber. The exhaust plume is used to calculate the flow characteristics of the nozzle. The test of grid sensitivity is conducted in all cases. In Case 3, adequate grid independence is satisfied with a mesh of 541419 cells, in which the minimum mesh volume and maximum mesh volume are about $8.489 \times 10^{-14} \mathrm{~m}^{3}$ and $2.952 \times 10^{-8} \mathrm{~m}^{3}$, respectively. Minimum orthogonal quality of mesh is 0.40 . Currently important, the grids near the wall, in the center of chamber and the throat are refined to ensure the $y^{+}$lower than 5 . The boundary conditions of the inlet and outlet are set as the mass flow inlet and the pressure outlet, respectively. Because the combustion residence time is short in the combustion chamber, the heat transfer through the chamber wall can be ignored, and the wall is considered as noslip, adiabatic wall in the simulation. And the pressure is given as $1 \mathrm{~atm}$ for the pressure outlet of exhaust plume and $28 \mathrm{~atm}$ for the mass flow inlet, respectively. Thus, the fluctuation of pressure is not considered.

In the simulations, the residuals of all components are lower $10^{-3}$. Concurrently important, the mass flow rate of the inlet, $49.975 \mathrm{~g} / \mathrm{s}$ is close to that of the outlet, $50.023 \mathrm{~g} / \mathrm{s}$. Therefore, the simulations are regarded as steady state solution.

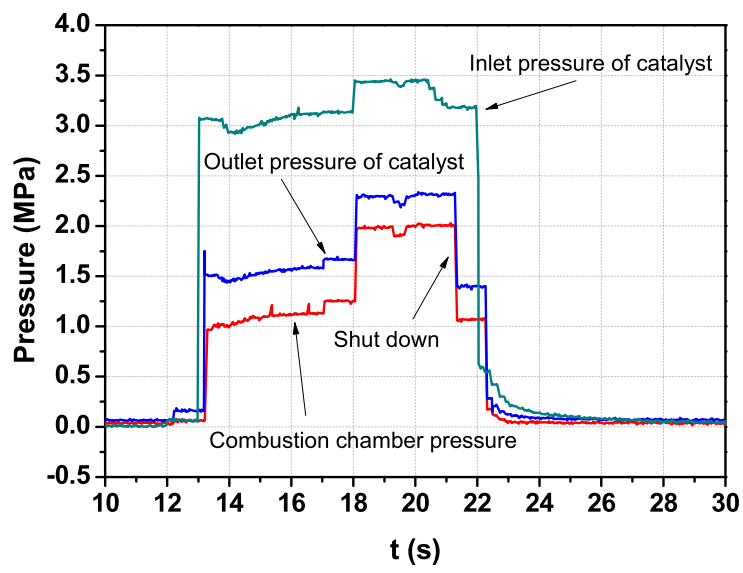

Fig. 5. The process of the hot-fire test.

\section{Results and discuss}

\subsection{Hot-fire testing}

Five co-axial swirl injectors are separately installed into the labscale engine. The operating pressures of the catalyst bed and combustion chamber are measured during the tests. In addition, the outlet temperature of the catalyst and the actual mass flow rate of $\mathrm{H}_{2} \mathrm{O}_{2}$ are measured. Fig. 5 depicts the pressure data measured by the pressure sensors. During the tests, $\mathrm{H}_{2} \mathrm{O}_{2}$ is injected $11 \mathrm{~s}$ before kerosene is injected, and the pressure of combustion chamber reaches $1 \mathrm{MPa}$ at that moment. Kerosene is injected at $17 \mathrm{~s}$, and the autoignition induces the chamber pressure reaches $2 \mathrm{MPa}$, thereby improving the inlet pressure and outlet pressure of catalyst, but the pressure drop of catalyst remains at a fixed value. The outlet temperature of the catalyst increases to over $600^{\circ} \mathrm{C}$. Some slight pressure perturbations still were observed in the steadystate operation; however, such perturbations are not relevant in obtaining the steady-state parameters. Because of the lack of cooling system, the hot-fire test lasts for approximately $4 \mathrm{~s}$ to avoid damaging the thruster.

Fig. 6 shows the evolution of the hydrogen peroxide mass flow rate measured by the Coriolis mass flowmeter. Between $12 \mathrm{~s}$ and $17 \mathrm{~s}$, the dashed field in Fig. 6 is induced by the false signal from the vibration of the opening valve. Subsequently, from $17 \mathrm{~s}$ to shut down, the average mass flow rate remains at a value of approximately $42 \mathrm{~g} / \mathrm{s}$, which is close to the design value. The uncertainty of mass flow is $0.035 \%$.

\subsection{Flow field analysis}

Five cases are simulated using CFD. To verify that the simulation results are reasonable, the simulation values are compared with the measured values, including the pressure of the combustion chamber, the geometric characteristic constant $A$ and the flow 


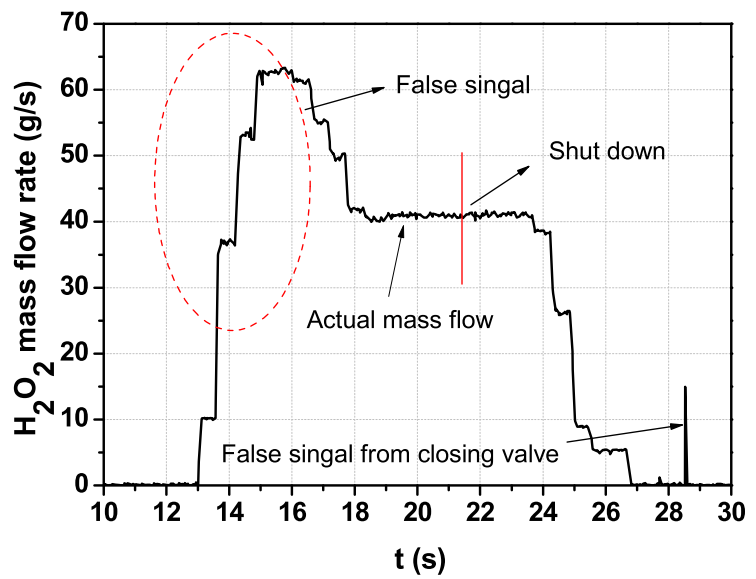

Fig. 6. The evolution of the $\mathrm{H}_{2} \mathrm{O}_{2}$ mass flow rate.

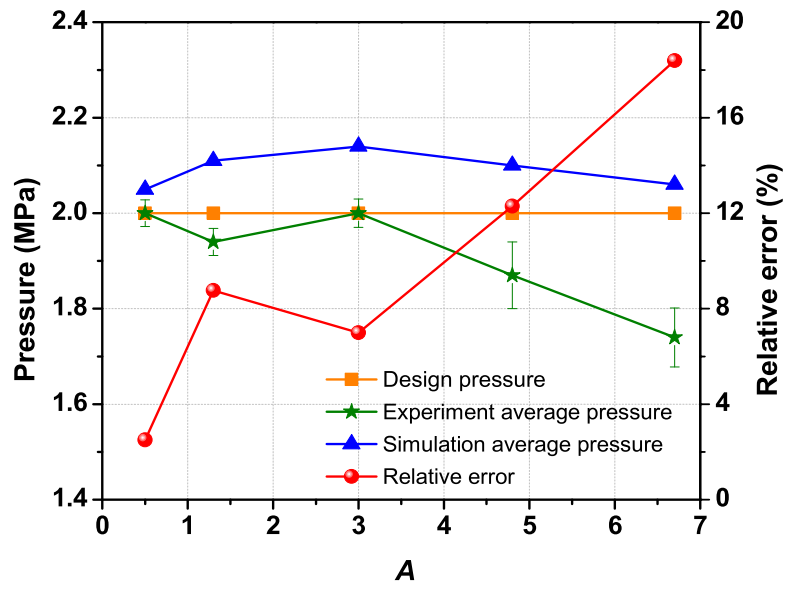

Fig. 7. The pressure of the experiment and the simulation.

characteristics of the nozzle. Case 3 is chosen as a comparison object, and the other cases exhibit similar results.

Fig. 7 shows comparison results of the average chamber pressure of five cases. One pressure sensor is installed in the inlet of combustion chamber. The pressure of the experiment is the average value of the pressure sensor with time. The pressure of simulation is the average value of the axial line. The average simulation values are higher than $2 \mathrm{MPa}$, and the maximal relative error is found for Case 1, 18.04\%. Thus, Case 1 is not considered a reliable one but is used as a reference. The adiabatic wall employed in the simulation is one of the main reasons for the higher pressure. Moreover, the pressure of the experiment is a time-averaged value, whereas the pressure of simulation is a steady-state value. Thus, high relative errors in the experiment are possible.

Fig. 8 depicts the results of the geometric characteristic constant $A$. The design value is obtained by using Eq. (3), and the simulation value is calculated using Eq. (4). In Eq. (4), $v_{\text {in }}$ and $v_{a}$ are substituted by the respective simulation area-weighted average values. The comparison of Fig. 8 proves that the geometry modeling of the gas swirl injector is reasonable, which reveals that the results are mainly affected by $A$ in the simulation.

The flow characteristics of the nozzle are calculated by the exhaust plume, which releases gases from the nozzle outlet. The atmospheric pressure is $1.036 \times 10^{5} \mathrm{~Pa}$ in the test, but the exit pressure of the nozzle is only $7.525 \times 10^{4} \mathrm{~Pa}$, as obtained from the simulation of case 3. According to the Laval nozzle, this situation can generate overexpansion. Thus, Fig. 9b shows the location of high static pressure in case 3. Because of high temperature ra-

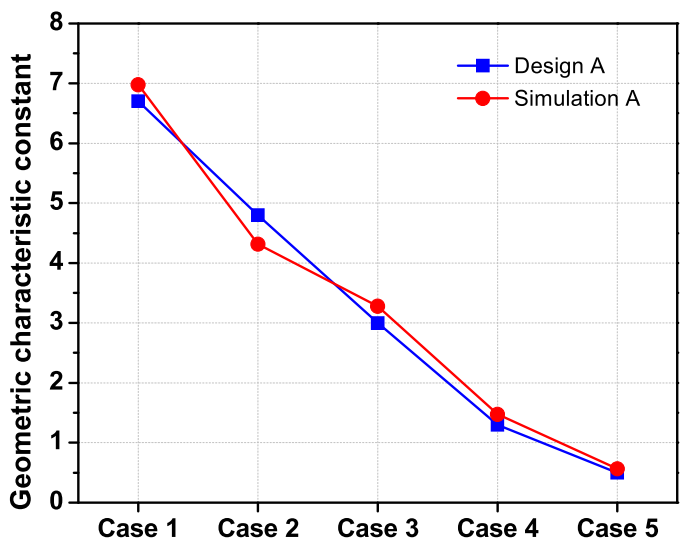

Fig. 8. The design and simulation of the geometric characteristic constant.

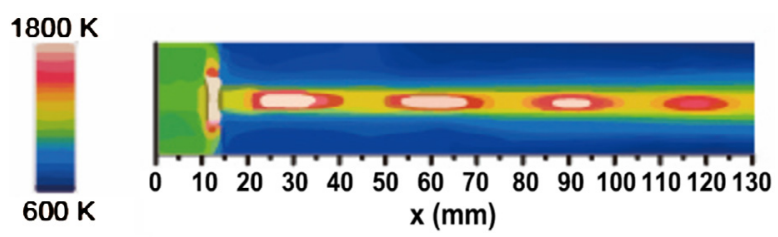

a. Mach disk image of the experiment

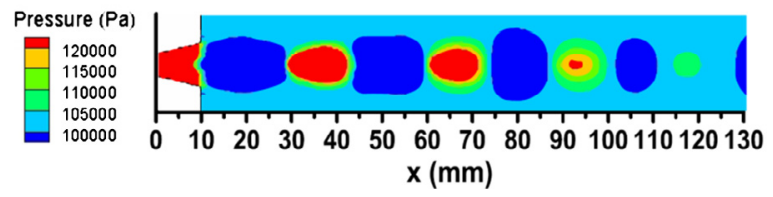

b. Static pressure contour of the simulation

Fig. 9. The location of Mach disks in the experiment and the simulation.

diation, a series of Mach disks, namely, the white color shades of image in Fig. 9a, can be captured by using a thermal infrared imager. In the plume zone, through aerodynamics, the location of high static pressure is at the location of high temperature. The location of the visible Mach disks (see Fig. 9a) and the location of high static pressure (see Fig. 9b) are similar in Fig. 9. The location of Mach disks is the characters of flow and combustion. And it is mainly decided by configuration of nozzle and adiabatic exponent. The latter is affected by combustion in chamber. Thus, the position of Mach dishes between simulations and experiments compare is compared, through which numerical methods are validated.

The temperature contours near the injector faceplate for the coaxial swirl injector and the coaxial jet injector are shown in Fig. 10. In the temperature contour from simulations, the length from inlet of combustion chamber to the zone of high temperature is defined as the flame length, $L_{c}$. The coaxial jet injector is designed and simulated in the same conditions, except for the different injection type.

From Fig. 10, the flame length of the coaxial jet injector is approximately $84 \mathrm{~mm}$. However, Fig. 11 indicates that the swirl injectors produce a far shorter flame length than the coaxial jet injector, and the flame length increases with the decrease of $A$. Meanwhile, the swirling flow yield centrifugal force that generates a low-pressure zone in the axial line of the inlet zone of chamber, and the high temperatures produced in the high-pressure zone are then rolled back into the low-pressure zone. This process induces the recirculation zone in the central line (Fig. 12). These results are similar to the results obtained by Cai's research [15]; i.e., the coaxial swirl injector has two recirculation zones in the simulation: one is located between the injector inlet and the chamber wall (called ORZ), and the other is located in the central line (called 


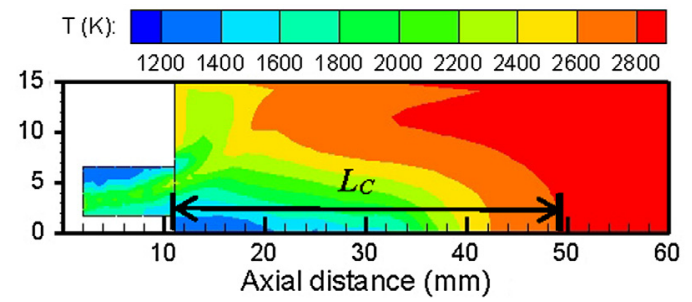

a. coaxial-swirl injector

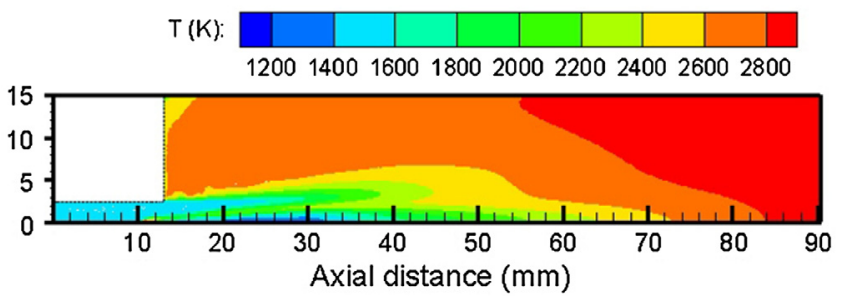

b. coaxial-jet injector

Fig. 10. The temperature contours near the injector faceplate from simulations.

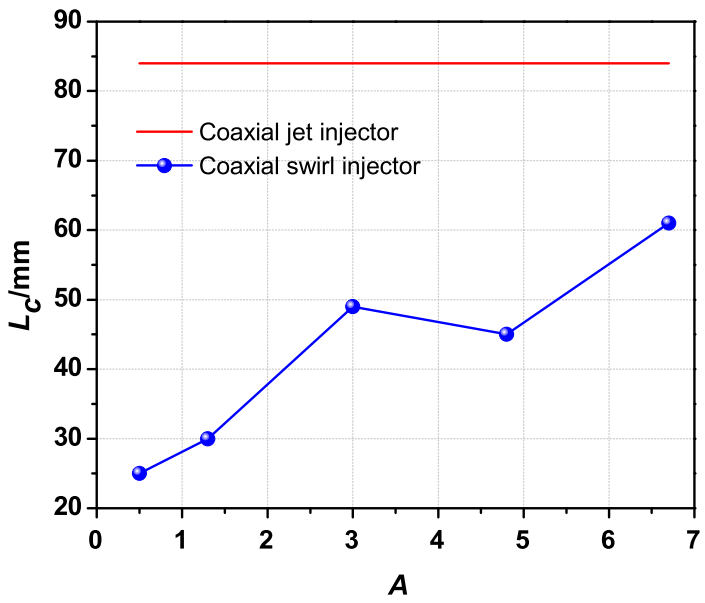

Fig. 11. Flame length of the coaxial-swirl injectors and the coaxial-jet injector.

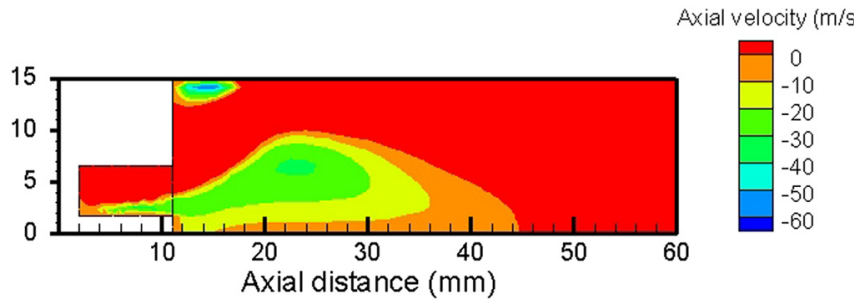

Fig. 12. Recirculation zone induced by swirling flow.

IRZ), which is crucial to the flame length and the combustion efficiency. Fig. 13a and Fig. 13b show that with a decreasing value of $A$, IRZ becomes thin and long. Thus, the mixing of oxidizer and fuel occurs more easily in the thin and long layer with a small $A$. Different from the coaxial shear flame length, the slight swirl flow helps make the flame area broad and mix the oxidizer with the fuel. Meanwhile, the inner recirculation zone (IRZ) becomes weak; thus, combustion occurs in the minor area, and the flame length becomes short. In contrast, the strong swirl flow causes the oxidizer to flow along the wall, which broadens the combustion area; however, the jet injector is in the centerline, and a small amount of fuel is entrained in the swirl flow. Thus, the flame length rises with the increase of $A$.

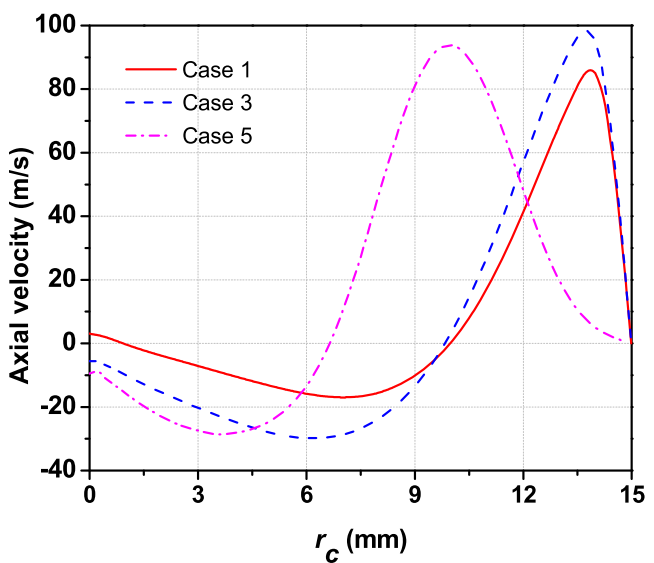

(a)

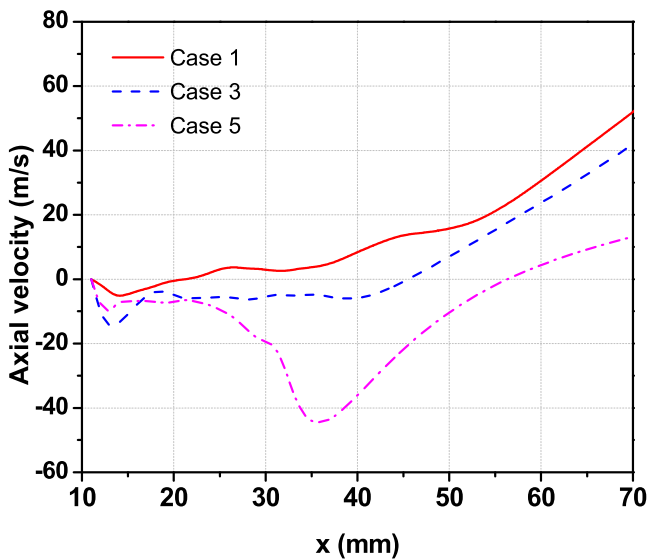

(b)

Fig. 13. (a) $x=12 \mathrm{~mm}$ axial velocity with radial distance. (b) Axial velocity with axial distance.

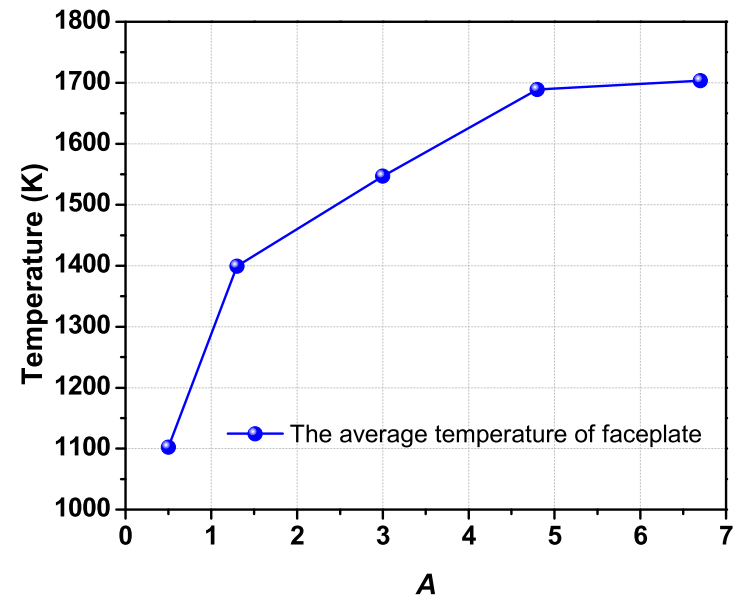

Fig. 14. The average temperature of faceplate in simulation.

The mass of the oxidizer flows with the chamber wall more easily with a large $A$, and the combustion occurs in the vicinity of the faceplate and chamber wall, inducing the faceplate of swirl injector yield high temperature. Thus, the average temperature of faceplate gradually rises with the increase of $A$ (Fig. 14). Moreover, Fig. 15 shows the five faceplates obtained after the experiment. It is obvious that the faceplates of Cases 1 and 2 have slight burns, whereas the other cases have bright faceplates. 


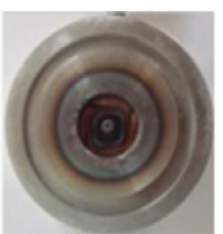

Case 1

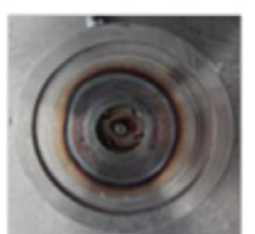

Case 2

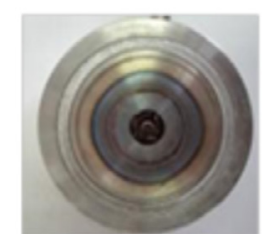

Case 3

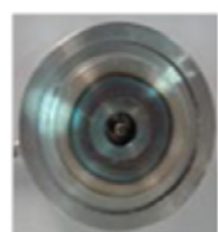

Case 4

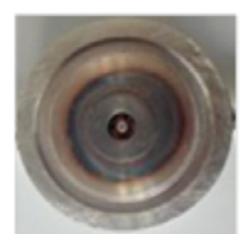

Case 5

Fig. 15. Five faceplate photos taken after the experiment.

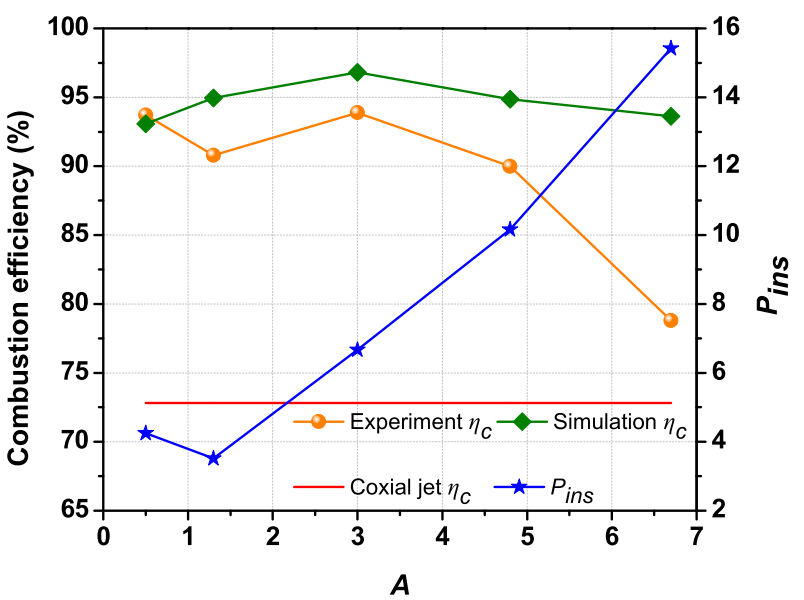

Fig. 16. Combustion efficiency and pressure instability of the $100-\mathrm{N}$-class thruster.

\subsection{Thrust chamber performance}

The characteristic velocity efficiency, $\left(\eta_{c}\right)$, which is a crucial factor to evaluate how the propellants are effectively combusted, is defined as

$\eta_{c}=\frac{C_{\text {exp }}^{*}}{C_{\text {ideal }}^{*}} \times 100 \%$

where $C_{\text {ideal }}^{*}$ is the ideal characteristic velocities and $C_{\text {exp }}^{*}$ is the experimental characteristic velocity. The former can be obtained from the NASA CEA code, and the $C_{\exp }^{*}$ values of the five cases are calculated using the following equation:

$C_{\exp }=\frac{P_{c} \bullet A_{t h}}{\dot{m}_{\text {tot }}}$

where $A_{t h}$ is the nozzle throat, $P_{c}$ denotes the average chamber pressure and $\dot{m}_{\text {tot }}$ represents the total mass flow rate of the propellants. Fig. 16 shows combustion efficiency in the experiment and the simulation as a function of geometric parameter $A$. It is obvious that Case 3 reaches the highest combustion efficiency. In addition, in the simulation results, with the value of $A$ increasing, the mass of the oxidizer flows more easily along the chamber wall, thus weakening the mixing effect; thus, the combustion efficiency reduces. However, a decrease in $A$ indicates that the tangential momentum of oxygen also decreases, which makes the mixing scheme close to that of the coaxial jet injector. The coaxial jet injector has a high axial velocity but thin interface with kerosene. Therefore, poor mixing results in a low combustion efficiency. Consequently, Cases 4 and 5 have low combustion efficiency.

The combustion instability was measured in terms of the pressure oscillation $\left(P_{\text {ins }}\right)$ inside the chamber [28], defined as

$P_{\text {ins }}=\frac{P_{\max }-P_{\min }}{P_{\text {ave }}} \times 100 \%$

where $P_{\max }$ and $P_{\min }$ are the maximum and minimum pressures during steady state test, respectively and $P_{\text {ave }}$ is the average chamber pressure during the steady state test. Fig. 16 indicates that the combustion instability is strengthened with the value of $A$ increasing. Tests from Ref. [28] indicate that the principal mode of combustion instability induced by coaxial injector mostly follows the first order tangential vibration mode. In addition, the tangential mode instability is predominant, particularly in the vicinity of the injector [29]. The pressure sensor of combustion chamber is installed near the injector. Thus, tangential momentum increasing may cause the tangential vibration more intensive, which may well be the reason why the combustion instability is intense with a large $A$. The instability requires more experimental studies to identify the main reasons. In addition, the pressure sensor should be installed far from the swirl injector.

\section{Conclusion}

This research was devoted to determining a feasible coaxial swirl injector to use in a $100-\mathrm{N}$-class $\mathrm{H}_{2} \mathrm{O}_{2} /$ kerosene thruster. An experiment and the CFD method were employed during the steady state ground test. The geometric parameter constant $A$ was shown to greatly affect the characteristics of the combustion flame and performance of thruster. The main results are summarized as follows.

(1) Due to centrifugal force, the swirl flow increases the mixing effect of the fuel and oxidizer, thus significantly reducing the flame length. Increasing the value of $A$ can help reduce the flame length at the expense of the heat conduction of the faceplate.

(2) A large or small value of $A$ is not conducive to improve the combustion efficiency. In the experiment, Case $3(A=3.0)$ reaches the highest combustion efficiency of $93.88 \%$. For the combustion stability, the pressure oscillation $\left(P_{\text {ins }}\right)$ inside the chamber gradually becomes more intense with an increase in $A$.

(3) For actual application, the pressure oscillation of Case 3 ( $A=$ $3.0), 6.67 \%$, is slightly higher than the recommended $5 \%$ during steady-state operation. However, overall, Case $3(A=3.0)$, has the optimal performance for $100-\mathrm{N}$-class $\mathrm{H}_{2} \mathrm{O}_{2} /$ kerosene thruster of the five cases considered. The $A$ between 1.3 and 3.0 may be more reasonable.

Future work will utilize more experimental measures to observe the flame structure of the swirl coaxial injector in a combustion chamber. In addition, the cooling equipment should be installed in a long-time steady state test, eventually realizing actual application.

\section{Conflict of interest statement}

There is no conflict of interest statement.

\section{Acknowledgements}

This work was supported by the National Natural Science Foundation of China (No. 91530112) and the Youth Innovation Foun- 


\section{dation of State Key Laboratory of High Temperature Gas Dynamics (No. LHD2016QN02).}

\section{References}

[1] R. Amri, D. Gibbon, T. Rezoug, The design, development and test of one newton hydrogen peroxide monopropellant thruster, Aerosp. Sci. Technol. 25 (2013) 266-272.

[2] P. Thakre, Y. Duan, V. Yang, Modeling of ammonium dinitramide (ADN) monopropellant combustion with coupled condensed and gas phase kinetics, Combust. Flame 161 (2014) 347-362.

[3] R. Yang, P. Thakre, V. Yang, Thermal decomposition and combustion of ammonium dinitramide (Review), Combust. Explos. Shock Waves 41 (2005) 657-679.

[4] P. Khare, V. Yang, H. Meng, G.A. Risha, R.A. Yetter, Thermal and electrolytic decomposition and ignition of HAN-water solutions, Combust. Sci. Technol 187 (7) (2015) 1065-1078.

[5] K. Anflo, T.A. Gronland, Development and testing of ADN-based monopropellants in small rocket engines, in: 36th AIAA/ASME/SAE/ASEE Joint Propulsion Conference \& Exhibit, 17-19 July 2000, pp. 17-19, AIAA 2000-3162.

[6] V.S. Kushneva, R.B. Gorshkova (Eds.), Spravochnik po toksikologii i gigienicheskim normativam potentsial'no opasnykh khimicheskikh veshchestv (Handbook in Toxicology and Hygienic Regulations of Potentially Dangerous Chemical Substances), Izdat, Moscow, 1999.

[7] Journal of the American College of Toxicology. Part B, Acute toxicity data, Mary Ann Liebert Inc., 1990.

[8] W.L. Li, P. Li, Y. Zou, Review and future trend of space propulsion technique using hydrocarbon propellants, J. Astron. 36 (3) (2015) 243-251 (in Chinese).

[9] Y. Moon, C. Park, S. Jo, S. Kwon, Design specifications of $\mathrm{H}_{2} \mathrm{O}_{2}$ / kerosene bipropellant rocket system for space missions, Aerosp. Sci. Technol. 33 (2014) $118-121$.

[10] A.S. Gohardani, J. Stanojev, A. Demairé, K. Anflo, M. Persson, N. Wingborg, C. Nilsson, Green space propulsion: opportunities and prospects, Prog. Aerosp. Sci. 71 (2014) 128-149.

[11] Y. Cong, T. Zhang, T. Li, J.W. Sun, X.D. Wang, L. Ma, D.B. Liang, L.W. Lin, Propulsive performance of a hypergolic $\mathrm{H}_{2} \mathrm{O}_{2}$ /kerosene bipropellant, J. Propuls. Power 20 (1) (2004) 83-86

[12] O.V. Romantsova, V.B. Ulybin, Safety issues of high-concentrated hydrogen peroxide production used as rocket propellant, Acta Astronaut. 109 (2015) 231-234.

[13] J.C. Sisco, B.L. Austin, J.S. Mok, W.E. Anderson, Autoignition of kerosene by decomposed hydrogen peroxide in a dump-combustor configuration, J. Propuls. Power 21 (3) (2005) 450-459.
[14] S. Li, Y.F. Ge, X.L. Wei, T. Li, Mixing and combustion modeling of hydrogen peroxide/kerosene shear-coaxial jet flame in lab-scale rocket engine, Aerosp. Sci. Technol. 56 (2016) 148-154.

[15] G.B. Cai, J. Dai, Y. Zhang, N.J. Yu, Combustion behaviors of GO2/GH2 swirl coaxial injector using non-intrusive optical diagnostics, Acta Astronaut. 123 (2016) 246-256.

[16] J. Dai, G.B. Cai, Y. Zhang, N.J. Yu, Experimental investigations of coaxial injectors in a laboratory-scale rocket combustor, Aerosp. Sci. Technol. 59 (2016) 41-51.

[17] V. Yang, M. Habiballah, J. Hulka, M. Popp, Liquid Rocket Thrust Chambers: Aspects of Modeling, Analysis, and Design, American Institute of Aeronautics and Astronautics Inc., 2004.

[18] Z.J. Wang, X.L. Chang, G. Tian, X.J. Zhang, The Design for Thrust Chamber of Liquid Propellant Rocket Engine, National Defense Industry Press, 2014 (in Chinese).

[19] J. Hulka, J.A. Schneider, Performance and stability of a booster class LOX/H2 swirl coaxial element injector, AIAA 1991-1877.

[20] J. Hulka, J.A. Schneider, Single element injector cold flow testing for STME swirl coaxial injector element design, AIAA 1993-2161.

[21] N. Zong, V. Yang, Supercritical fluid dynamics of pressure swirl injector with external excitations, in: 43rd AIAA/ASME/SAE/ASEE Joint Propulsion Conference \& Exhibit, 8-11 July 2007, AIAA 2007-5458.

[22] G.C. Zhang, Production Techniques of Hydrogen Peroxide, Chemistry Industry Press, 2012 (in Chinese).

[23] F.Q. Zhong, X.J. Fan, G. Yu, J.G. Li, Heat transfer of aviation kerosene at supercritical conditions, J. Thermophys. Heat Transf. 23 (3) (2009) 543-550.

[24] L.J. Yang, Q.F. Fu, The Design of Liquid Rocket Engine Thrust, Beijing University of Aeronautics and Astronautics Press, 2013 (in Chinese).

[25] J.O. Valderrama, The state of the cubic equations of state, Ind. Eng. Chem. Res. 42 (8) (2003) 1603-1618.

[26] D. Zheng, W.M. Yu, B.J. Zhong, RP-3 aviation kerosene surrogate fuel and the chemical reaction kinetic model, Acta Physico-Chimica Sinica 31 (4) (2015) 636-642.

[27] J.Q. Xu, J.J. Guo, A.K. Liu, J.L. Wang, N.X. Tan, X.Y. Li, Construction of autoignition mechanisms for the combustion of RP-3 surrogate fuel and kinetics simulation, Acta Physico-Chimica Sinica 31 (4) (2015) 643-652.

[28] H. Kang, D. Jang, S. Kwon, Demonstration of 500 N scale bipropellant thruster using non-toxic hypergolic fuel and hydrogen peroxide, Aerosp. Sci. Technol. 49 (2016) 209-214.

[29] G.P. Sutton, O. Biblarz, Rocket Propulsion Elements, 8th edition, John Wiley \& Sons Inc., 2010. 